\title{
A potential-free field inverse time-fractional Schrödinger problem: optimal error bound analysis and regularization method
}

\author{
Fan Yang ${ }^{1}$, Junliang $\mathrm{Fu}^{2}$, and Xiao-Xiao $\mathrm{Li}^{1}$ \\ ${ }^{1}$ LanZhou University of Technology \\ ${ }^{2}$ Lanzhou University of Technology
}

April 27, 2020

\begin{abstract}
In this paper, an inverse time-fractional Schr\$ $\ \operatorname{ddot}\{0\} \$$ dinger problem of potential-free field is studied. This problem is illposed, i.e, the solution (if it exists) does not depend continuously on the data. Based on an a priori bound condition, the optimal error bound analysis is given. Moreover, a modified kernel method is introduced. The convergence error estimate obtained by this method under the priori regularization parameter selection rule is optimal, and the convergence error estimate obtained under the posteriori regularization parameter selection rule is order-optimal. Finally, some numerical examples are given to illustrate the effectiveness and stability of this method.
\end{abstract}

Hosted file 1DfractionalSDR.pdf available at https://authorea.com/users/315227/articles/445592-a-potentialfree-field-inverse-time-fractional-schr

Hosted file picture.rar available at https://authorea.com/users/315227/articles/445592-a-potential-free-fieldinverse-time-fractional-schr 